\title{
CONHECIMENTO DA EQUIPE DE ENFERMAGEM SOBRE LESÃO POR PRESSÃO
}

\author{
Jaqueline Aparecida dos Santos Sokem 1,* (D), Elaine Aparecida Mye Takamatu Watanabe² (D, \\ Adriano Menis Ferreira ${ }^{3}$ (D), Lillian Dias Castilho Siqueira ${ }^{4}$ (B), Manuela de Mendonça \\ Figueirêdo Coelho ${ }^{5}$ (D), Fabiana Perez Rodrigues Bergamaschi² (D)
}

\begin{abstract}
RESUMO
Objetivo: avaliar o nível de conhecimentos da equipe de enfermagem da clínica médica de um hospital universitário sobre lesão por pressão. Método: estudo descritivo-exploratório, aprovado por Comitê de Ética em Pesquisa. Os dados foram coletados através de questionário validado. Participaram 50 indivíduos, sendo 35 técnicos de enfermagem e 15 enfermeiros. Resultados: os técnicos em enfermagem obtiveram média de 83,5\% e os enfermeiros obtiveram média de 89,9\%. Realizou-se um modelo de regressão para verificar variáveis que influenciam no nível de conhecimento, sendo identificado que possuir mais de 5 anos na profissão aumenta 1,61 vezes a chance de o profissional apresentar um conhecimento adequado. Conclusão: foi identificada uma fragilidade no conhecimento sobre lesão por pressão. Tendo em vista os custos envolvidos no tratamento e as complicações advindas dessas lesões, as instituições de saúde devem implementar ações educativas sobre esse agravo.
\end{abstract}

DESCRITORES: Lesão por pressão. Cuidados de enfermagem. Segurança do paciente. Estomaterapia.

\section{NURSING TEAM KNOWLEDGE ABOUT PRESSURE INJURIES}

\begin{abstract}
Objective: to assess the level of knowledge of the nursing staff of the medical clinic of a university hospital about pressure injuries. Method: descriptive-exploratory study, approved by the Research Ethics Committee. Data were collected through a validated questionnaire. Fifty individuals participated, 35 nursing technicians and 15 nurses. Results: nursing technicians had an average of $83.5 \%$ and nurses had an average of $89.9 \%$. A regression model was carried out to verify variables that influence the level of knowledge, and it was identified that having more than 5 years in the profession increases 1.61 times the chance that the professional has adequate knowledge. Conclusion: a weakness in knowledge about pressure injuries was identified. In view of the costs involved in the treatment and complications arising from these injuries, health institutions must implement educational actions about this condition.
\end{abstract}

DESCRIPTORS: Pressure injury. Nursing care. Patient safety. Stomatherapy.

1. Universidade Federal da Grande Dourados - Hospital Universitário - Unidade de Clínica Médica - Dourados (MS), Brasil.

2. Universidade Estadual do Mato Grosso do Sul - Centro de Ciências Agrárias, Biológicas e da Saúde - Departamento de Enfermagem - Dourados (MS), Brasil.

3. Universidade Federal do Mato Grosso do Sul - Centro de Ciências Biológicas e da Saúde - Departamento de Enfermagem Três Lagoas (MS), Brasil.

4. Universidade Federal da Grande Dourados - Hospital Universitário - Setor de Gestão da Pesquisa e Inovação Tecnológica Dourados (MS), Brasil.

5. Universidade Federal do Ceará - Centro de Ciências da Saúde - Departamento de Enfermagem - Fortaleza (CE), Brasil.

*Autor correspondente: jaqueline_skm@hotmail.com

Editor de Seção: Juliano Teixeira Moraes

Recebido: Set. 03, 2021 | Aceito: Nov. 01, 2021

Como citar: Sokem JAS; Watanabe EAMT; Ferreira AM; Siqueira LDC; Coelho MMF; Bergamaschi FPR. Conhecimento da equipe de enfermagem sobre a lesão por pressão. ESTIMA, Braz. J. Enterostomal Ther., 2021, 19: e2521. https://doi.org/10.30886/ estima.v19.1129_PT 


\section{CONOCIMIENTOS DEL EQUIPO DE ENFERMERÍA SOBRE LA LESIÓN \\ POR PRESIÓN}

\section{RESUMEN}

Objetivo: evaluar el nivel de conocimientos del equipo de enfermería de clínica médica de un hospital universitario sobre lesión por presión. Método: estudio descriptivo/exploratorio, aprobado por Comité de Ética en Investigación. Los datos fueron recolectados por medio de un cuestionario validado. Participaron 50 individuos, siendo 35 técnicos de enfermería y 15 enfermeros. Resultados: los técnicos en enfermería obtuvieron un promedio del 83,5 \% y los enfermeros un promedio del 89,9\%. Se realizó un modelo de regresión para verificar variables que influyen en el nivel de conocimiento, identificándose que el contar con más de 5 años en la profesión aumenta 1,61 veces la probabilidad que el profesional presente un conocimiento adecuado. Conclusión: fue identificada una fragilidad en el conocimiento sobre lesión por presión. Teniendo en cuenta los costos involucrados en el tratamiento y las complicaciones derivadas de esas lesiones, las instituciones de salud deben implementar acciones educativas sobre esa afección.

DESCRIPTORES: Lesión por presión. Cuidados de enfermería. Seguridad del paciente. Estomaterapia.

\section{INTRODUÇÃO}

Nos últimos anos a assistência hospitalar passou a ser mais complexa devido à crescente inovação tecnológica, às inúmeras atividades desempenhadas pelos profissionais, além da presença de interrupções no cuidado aos pacientes. Nessa perspectiva, uma assistência insegura resulta em morbidade e mortalidade evitáveis, com indivíduos mais vulneráveis às complicações como, por exemplo, a lesão por pressão (LP) $)^{1,2}$.

A LP é avaliada como um indicador negativo da qualidade da assistência em saúde, sendo definida como um dano localizado na pele e/ou nos tecidos moles subjacentes sobre uma proeminência óssea. A LP pode também estar relacionada ao uso de dispositivo médico ou outro artefato. É compreendida como um evento adverso e representa importante desafio assistencial, uma vez que gera sofrimento físico e psicológico para pacientes e familiares, além de elevados custos financeiros às instituições ${ }^{3,4}$.

Estudo conduzido em um hospital universitário de Minas Gerais identificou uma frequência de LP de 29\% na unidade de clínica médica, $16 \%$ na clínica cirúrgica e 53,8\% na observação do pronto socorro. Já no cenário internacional, pesquisa com objetivo de analisar a prevalência e as características de LP relacionadas ao uso de dispositivos médicos em pacientes provenientes de instituições canadenses e norte-americanas identificou que a prevalência geral foi de $7,2 \%$, e a prevalência adquirida nas instituições foi de $3,1 \%$,

As recomendações para prevenção e tratamento de feridas crônicas envolvem a avaliação sistematizada do paciente e sua ferida, o controle dos fatores causais e associados e o preparo do leito da ferida, elementos que exigem constante atualização dos enfermeiros?

Nesse contexto, pesquisadores elaboraram diretrizes internacionais relacionadas à prevenção e tratamento da LP, considerando, entre as orientações e condutas sobre essa lesão, recomendações para a implementação de políticas públicas, educação de profissionais e pesquisas sobre a temática. No entanto, apesar dessas diretrizes, estudos apontam deficiências no conhecimento dos profissionais, além de elevadas taxas de incidência e prevalência de LP nos serviços de saúde e domicílios ${ }^{8-10}$.

Dentre os diversos profissionais que compõem a equipe multidisciplinar, a equipe de enfermagem permanece por maior tempo em contato com os pacientes nas instituições de saúde. Em cenários clínicos, possuem papel fundamental na tomada de ações preventivas. Daí a importância da manutenção de níveis adequados de conhecimento e atitudes assertivas por parte desses profissionais. A utilização de práticas de cuidado pautadas em uma enfermagem baseada em evidências favorece a diminuição do tempo de permanência hospitalar e a redução de eventos adversos ${ }^{11,12}$.

Diante do exposto e considerando a constante necessidade de diagnosticar o grau de conhecimento dos profissionais de saúde sobre LP, bem como a escassez de publicações sobre esse tema na região Centro-Oeste do Brasil, justifica-se o objetivo do artigo em tela: avaliar o nível de conhecimentos da equipe de enfermagem da clínica médica de um hospital universitário sobre LP. 


\section{MÉTODO}

Estudo descritivo e exploratório, de abordagem quantitativa quanto ao tratamento dos dados, conduzido no período de agosto a setembro de 2017, realizado no Hospital Universitário da Universidade Federal da Grande Dourados / Empresa Brasileira de Serviços Hospitalares (HU-UFGD/EBSERH), com a equipe de enfermagem da clínica médica. A instituição onde foi realizada a pesquisa é de média complexidade e está localizada no estado de Mato Grosso do Sul, sendo referência para 35 municípios, incluindo a população indígena e de fronteira.

Os aspectos éticos foram respeitados em consonância com a resolução no 466/2012. O projeto foi aprovado pelo Comitê de Ética em Pesquisa da Universidade Estadual de Mato Grosso do Sul, sob o parecer no 2.197.369 e CAAE 70593017.8.0000.8030. Todos os participantes assinaram o Termo de Consentimento Livre e Esclarecido e permaneceram com uma via em sua posse.

O quadro total de profissionais da enfermagem na unidade era de 57 técnicos de enfermagem e 15 enfermeiros $(\mathrm{n}=72)$. Foram convidados a participar todos os profissionais presentes durante o período da coleta de dados. Optou-se por amostra não probabilística por conveniência devido às características do setor em que as LP são prevalentes. Os critérios de inclusão foram: atuar diretamente na assistência a pacientes na unidade e aceitar participar voluntariamente do estudo. Os critérios de exclusão foram: a ausência do servidor devido às férias, licença médica ou folga.

Para a coleta das informações, primeiramente, utilizou-se um instrumento elaborado pelos autores deste estudo, com o intuito de coletar os dados sociodemográficos dos profissionais. Quanto à coleta de dados referente ao nível de conhecimento, foi utilizado o Teste de Pieper. O referido teste apresenta 41 afirmações com itens relacionados à prevenção, avaliação e classificação da $\mathrm{LP}^{13}$. Esse teste foi traduzido e validado em estudo realizado no Brasil ${ }^{14}$. À época da coleta de dados (2017), esse era o instrumento disponível no Brasil para avaliação de conhecimentos de LP, contudo, em 2018, outro instrumento com essa finalidade foi traduzido, adaptado transculturalmente e validado na língua portuguesa brasileira ${ }^{4}$.

Para a presente pesquisa, o questionário sofreu adaptações devido à atualização da literatura acerca do tema, que serão descritas a seguir: mudança do termo úlcera por pressão para lesão por pressão; modificação no item 8, retirando a indicação de uso do amido de milho e acrescentando a cobertura de espuma de poliuretano para prevenção da fricção, e alteração no item 26, em que foi modificado o termo Unidade de Terapia Intensiva para setor, visto que a aplicação do instrumento ocorreu em uma unidade de clínica médica ${ }^{8}$. As alterações não passaram por processo de validação, contudo, a utilização do instrumento e as modificações foram autorizadas pela autora.

O instrumento foi aplicado de maneira individual, durante o turno de trabalho, sendo devolvido em envelope para a pesquisadora, sem identificação, de modo a assegurar o anonimato dos participantes.

Os dados foram digitados e conferidos através de dupla digitação. Posteriormente, foi realizada a análise estatística através do programa estatístico Statistical Package for Social Science (SPSS), versão 22.0. Realizou-se análise estatística descritiva e testes estatísticos para comparação das possíveis variáveis. Para a verificação de possíveis diferenças entre os escores de acertos entre as categorias profissionais, aplicou-se o teste t de Student e o teste Mann-Whitney. Ao considerar o conhecimento adequado representado pelo percentual de acertos de $90 \%$ dos itens, dicotomizou-se o nível de conhecimento dos profissionais em adequado (média maior ou igual que 36,9) e não adequado (média menor que 36,9$)^{14}$.

Foi realizada uma regressão logística binária (método enter) para identificar se as variáveis tempo de trabalho (maior ou igual a 5 anos), ter treinamento prévio sobre LP e Dermatite Associada à Incontinência (DAI) (sim ou não) e o cargo (enfermeiro ou técnico) seriam preditoras para o desfecho conhecimento adequado. Adotou-se o nível de significância de $5 \%$.

\section{RESULTADOS}

Compuseram o estudo 50 profissionais de enfermagem. Desses, 35 eram técnicos de enfermagem e 15 enfermeiros. A amostra caracterizou-se por idade predominante de 30 a 39 anos (46\%), seguida dos 20 a 29 anos (22\%); sexo feminino (56\%) e tempo de profissão entre 5 e 9 anos e 15 e 19 anos (ambos 26\%). Com relação ao nível de escolaridade, dos profissionais, (62\%) apresentava graduação e (46\%) especialização. 
Considerando as médias de acertos globais das 41 questões, os técnicos em enfermagem obtiveram média de (83,5\%) e os enfermeiros obtiveram média de (89,9\%). Os itens sobre a avaliação e classificação da LP estão descritos na Tabela 1.

Tabela 1. Frequência de acertos dos participantes no teste, segundo os itens sobre avaliação e classificação da lesão por pressão. Dourados (MS), Brasil - 2017.

\begin{tabular}{|c|c|c|c|}
\hline Questões avaliadas & $\begin{array}{c}\text { Técnico de } \\
\text { enfermagem } \\
N(\%)\end{array}$ & $\begin{array}{c}\text { Enfermeiro } \\
\mathrm{N}(\%)\end{array}$ & $\begin{array}{l}\text { Total } \\
\text { N (\%) }\end{array}$ \\
\hline 1 - O estágio I da LP é definido como um eritema que não embranquece (V) & $35(100,0)$ & $14(93,3)$ & $48(96,0)$ \\
\hline 6 - Uma LP em estágio III é uma perda parcial de pele envolvendo a epiderme (F) & $32(91,4)$ & $15(100,0)$ & $47(94,0)$ \\
\hline $\begin{array}{l}9 \text { - LP no estágio IV apresentam uma perda de pele total com intensa destruição } \\
\text { e necrose tissular ou danos aos músculos, ossos ou estruturas de suporte }(V)\end{array}$ & $34(97,1)$ & $15(100,0)$ & $49(98,0)$ \\
\hline 20 - LP no estágio II apresentam uma perda da pele na sua espessura total (F) & $17(48,6)$ & $10(66,6)$ & $27(54,0)$ \\
\hline 31 - As LP são feridas estéreis (F) & $31(88,6)$ & $14(93,3)$ & $45(90,0)$ \\
\hline 32 - Uma cicatriz de LP poderá lesar mais rapidamente que a pele íntegra (V) & $33(94,3)$ & $15(100,0)$ & $48(96,0)$ \\
\hline 33 - Uma bolha no calcâneo não deve ser motivo de preocupação (F) & $34(97,1)$ & $15(100,0)$ & $49(98,0)$ \\
\hline $\begin{array}{l}38 \text { - As LP em estágio II podem ser extremamente dolorosas pela exposição de } \\
\text { terminações nervosas }(V)\end{array}$ & $21(60,0)$ & $12(80,0)$ & $33(66,0)$ \\
\hline
\end{tabular}

LP = Lesão por Pressão; $V$ = Verdadeiro; F= Falso; $N$ = Frequência.

A maior taxa de acertos na categoria avaliação e classificação da LP foi obtida pelos enfermeiros nos itens 6, 9, 32 e 33, com 100\% de acerto. Os mesmos profissionais obtiveram uma menor taxa de acertos no item 20. Em relação aos técnicos, esses alcançaram elevada taxa de acertos nos itens 1, 9 e 33; também apresentaram menor média no item 20.

Considerando o total de respondentes, verificou-se uma fragilidade no conhecimento sobre classificação das LP de estágio II (item 20) e na questão referente à presença de dor na LP de estágio II (item 38). Os itens referentes à prevenção da LP estão descritos na Tabela 2.

Tabela 2. Frequência de acertos dos participantes no teste, segundo os itens sobre prevenção de lesão por pressão. Dourados (MS), Brasil - 2017.

\begin{tabular}{|c|c|c|c|}
\hline Questões avaliadas & $\begin{array}{l}\text { Técnico de } \\
\text { enfermagem } \\
\mathrm{N}(\%)\end{array}$ & $\begin{array}{l}\text { Enfermeiro } \\
\qquad \mathrm{N}(\%)\end{array}$ & $\begin{array}{l}\text { Total } \\
\mathrm{N}(\%)\end{array}$ \\
\hline $\begin{array}{l}2 \text { - São fatores de risco para o desenvolvimento da LP: mobilidade; } \\
\text { incontinência; nutrição adequada e alteração do nível de consciência (V) }\end{array}$ & $31(88,6)$ & $14(93,3)$ & $45(90,0)$ \\
\hline $\begin{array}{l}3 \text { - Todos os indivíduos de risco para LP devem ter uma inspeção sistemática } \\
\text { da pele pelo menos uma vez por semana (F) }\end{array}$ & $35(100,0)$ & $15(100,0)$ & $50(100,0)$ \\
\hline $\begin{array}{l}4 \text { - Água quente e sabonete podem ressecar a pele e aumentar o risco } \\
\text { para LP (V) }\end{array}$ & $24(68,6)$ & $11(73,3)$ & $35(70,0)$ \\
\hline $\begin{array}{l}5 \text { - É importante massagear as proeminências ósseas se estiverem } \\
\text { hiperemiadas (F) }\end{array}$ & $17(48,6)$ & $12(80,0)$ & $29(58,0)$ \\
\hline $\begin{array}{l}7 \text { - Todo indivíduo, na admissão hospitalar, deve ser avaliado quanto ao risco } \\
\text { para desenvolver LP }(V)\end{array}$ & $35(100,0)$ & $15(100,0)$ & $50(100,0)$ \\
\hline $\begin{array}{l}8 \text { - Cremes, curativos transparentes (tipo Tegaderm ou Opsite), curativos de } \\
\text { espuma de poliuretano e/ou hidrocoloides (tipo Duoderm) não protegem } \\
\text { contra os efeitos da fricção (F) }\end{array}$ & $28(80,0)$ & $15(100,0)$ & $43(86,0)$ \\
\hline $\begin{array}{l}10 \text { - Uma ingestão dietética adequada às necessidades de proteínas e calorias } \\
\text { do paciente deve ser mantida durante a doença }(V)\end{array}$ & $35(100,0)$ & $15(100,0)$ & $50(100,0)$ \\
\hline $\begin{array}{l}11 \text { - As pessoas que ficam restritas ao leito devem ser reposicionadas a cada } \\
3 \text { horas (F) }\end{array}$ & $28(80,0)$ & $15(100,0)$ & $43(86,0)$ \\
\hline
\end{tabular}


Tabela 2. Continuação...

\begin{tabular}{|c|c|c|c|}
\hline Questões avaliadas & $\begin{array}{l}\text { Técnico de } \\
\text { enfermagem } \\
\mathrm{N}(\%)\end{array}$ & $\begin{array}{l}\text { Enfermeiro } \\
\qquad \mathrm{N}(\%)\end{array}$ & $\begin{array}{l}\text { Total } \\
\mathrm{N}(\%)\end{array}$ \\
\hline $\begin{array}{l}12 \text { - Uma escala com horários para mudança de decúbito deveria ser escrita } \\
\text { para cada paciente }\end{array}$ & $32(91,4)$ & $13(86,7)$ & $45(90,0)$ \\
\hline 13 - Protetores como luvas d'água aliviam a pressão nos calcâneos (F) & $12(34,3)$ & $11(73,3)$ & $23(46,0)$ \\
\hline 14 - Rodas d'água ou almofadas tipo argola auxiliam na prevenção da LP (F) & $10(28,6)$ & $12(80,0)$ & $22(44,0)$ \\
\hline $\begin{array}{l}15 \text { - Na posição lateral, a pessoa deve ficar em ângulo de } 30 \text { graus com } \\
\text { a cama }(V)\end{array}$ & $27(77,1)$ & $12(80,0)$ & $39(78,0)$ \\
\hline $\begin{array}{l}16 \text { - A cabeceira da cama deve ser mantida em um baixo grau de elevação } \\
\text { (não maior que um ângulo de } 30 \text { graus), consistente com as condições clínicas } \\
\text { e recomendações médicas ( } V \text { ) }\end{array}$ & $15(42,8)$ & $07(46,7)$ & $22(44,0)$ \\
\hline $\begin{array}{l}17 \text { - Uma pessoa que não pode se movimentar deve ser reposicionada, } \\
\text { enquanto sentada na cadeira, a cada } 2 \text { horas (F) }\end{array}$ & $11(31,4)$ & $03(20,0)$ & $14(28,0)$ \\
\hline $\begin{array}{l}18 \text { - As pessoas que podem aprender devem ser orientadas a mudar seu peso } \\
\text { a cada } 15 \text { minutos enquanto sentadas na cadeira }(V)\end{array}$ & $28(80,0)$ & $11(73,3)$ & $39(78,0)$ \\
\hline $\begin{array}{l}19 \text { - As pessoas que permanecem na cadeira devem usar uma almofada para } \\
\text { proteção no assento }(V)\end{array}$ & $31(88,6)$ & $12(80,0)$ & $43(86,0)$ \\
\hline 21 - A pele deve permanecer limpa e seca (V) & $35(100,0)$ & $15(100,0)$ & $50(100,0)$ \\
\hline $\begin{array}{l}22 \text { - Medidas de prevenção não necessitam ser usadas para prevenir novas } \\
\text { lesões quando o paciente já possui LP (F) }\end{array}$ & $29(82,9)$ & $15(100,0)$ & $44(88,0)$ \\
\hline $\begin{array}{l}23 \text { - Lençol móvel ou forros devem ser utilizados para transferir ou } \\
\text { movimentar pacientes }(V)\end{array}$ & $35(100,0)$ & $14(93,3)$ & $49(98,0)$ \\
\hline $\begin{array}{l}24 \text { - A mobilização e a transferência de pacientes totalmente dependentes } \\
\text { devem ser feitas por duas ou mais pessoas }(V)\end{array}$ & $34(97,1)$ & $15(100,0)$ & $49(98,0)$ \\
\hline $\begin{array}{l}25 \text { - Medidas de reabilitação devem ser instituídas se o estado geral do } \\
\text { paciente permitir }(V)\end{array}$ & $33(94,3)$ & $12(80,0)$ & $45(90,0)$ \\
\hline $\begin{array}{l}26 \text { - Todo paciente admitido no setor deve ser submetido à avaliação do risco } \\
\text { para o desenvolvimento de LP (V) }\end{array}$ & $33(94,3)$ & $15(100,0)$ & $48(96,0)$ \\
\hline $\begin{array}{l}27 \text { - Pacientes e familiares devem ser orientados quanto às causas e fatores de } \\
\text { risco para o desenvolvimento de LP (V) }\end{array}$ & $35(100,0)$ & $15(100,0)$ & $50(100,0)$ \\
\hline 28 - As proeminências ósseas devem ficar em contato direto uma com a outra (F) & $35(100,0)$ & $15(100,0)$ & $50(100,0)$ \\
\hline $\begin{array}{l}29 \text { - Toda pessoa avaliada como em risco para desenvolver LP deveria ser } \\
\text { colocada em colchão redutor de pressão (V) }\end{array}$ & $29(82,9)$ & $12(80,0)$ & $41(82,0)$ \\
\hline 30 - A pele exposta à umidade danifica-se mais facilmente $(V)$ & $34(97,1)$ & $15(100,0)$ & $49(98,0)$ \\
\hline 34 - Uma boa maneira de diminuir a pressão nos calcâneos é elevá-los do leito (V) & $34(97,1)$ & $13(86,7)$ & $47(94,0)$ \\
\hline $\begin{array}{l}35 \text { - Todo o cuidado administrado para prevenir ou tratar LP não precisa ser } \\
\text { documentado (F) }\end{array}$ & $32(91,4)$ & $15(100,0)$ & $47(94,0)$ \\
\hline $\begin{array}{l}36 \text { - Cisalhamento é a força que ocorre quando a pele adere a uma superfície } \\
\text { e o corpo desliza em sentido contrário }(V)\end{array}$ & $27(77,1)$ & $15(100,0)$ & $42(84,0)$ \\
\hline 37 - A fricção pode ocorrer ao movimentar a pessoa na cama (V) & $31(88,6)$ & $14(93,3)$ & $45(90,0)$ \\
\hline $\begin{array}{l}39 \text { - Para as pessoas que têm incontinência, a limpeza da pele deve ocorrer no } \\
\text { momento que se sujar e nos intervalos de rotina (V) }\end{array}$ & $35(100,0)$ & $14(93,3)$ & $49(98,0)$ \\
\hline 40 - Programas educacionais podem reduzir a incidência de LP (V) & $35(100,0)$ & $15(100,0)$ & $50(100,0)$ \\
\hline $\begin{array}{l}41 \text { - Pacientes hospitalizados precisam ser avaliados quanto ao risco para LP } \\
\text { uma única vez (F) }\end{array}$ & $34(97,1)$ & $15(100,0)$ & $49(98,0)$ \\
\hline
\end{tabular}

LP = Lesão por pressão; $V$ = verdadeiro; $F$ = falso; $N$ = frequência.

Quanto à comparação no desempenho dos respondentes, foi realizado o teste estatístico de Mann-Whitney e o teste t para amostras independentes, para essa finalidade. Esses dados estão apresentados na Tabela 3. 
Tabela 3. Comparação dos acertos entre os enfermeiros e os técnicos de enfermagem, segundo as dimensões e totalidade do instrumento. Dourados (MS), Brasil - 2017.

\begin{tabular}{lccc}
\hline \multicolumn{1}{c}{ Dimensão do instrumento } & \multicolumn{3}{c}{ Média de acertos } \\
\cline { 2 - 4 } & Enfermeiros & Técnicos & p \\
\hline Avaliação e classificação da lesão por pressão & 33,32 & 21,67 & $0,06 *$ \\
\hline Prevenção de lesão por pressão & 30,69 & 19,88 & $0,01 *$ \\
\hline Todas as questões & 36,83 & 34,22 & 0,01 ** \\
\hline
\end{tabular}

* Teste Mann-Whitney; ** Teste t para amostras independentes.

Os enfermeiros apresentaram maiores escores em relação às dimensões avaliação e classificação $(U=128,500, z=-2,743$, $\mathrm{p}=0,006)$ e prevenção $(U=108,000, z=-2,529, \mathrm{p}=0,011)$, bem como apresentaram escores estatisticamente maiores (Média=36,8; DP=1,586) do que os técnicos (Média=34,22; DP=3,200) $(t(42)=2,694, \mathrm{p}=0,10)$, evidenciando que os enfermeiros têm um nível de conhecimento maior que o dos técnicos.

Ao verificar se os níveis de conhecimento poderiam ser influenciados pelos fatores tempo de trabalho, existência de treinamento prévio sobre LP e DAI e o cargo, obteve-se o modelo abaixo descrito na Tabela 4.

Tabela 4. Modelo de regressão com variáveis preditoras de conhecimento adequado. Dourados (MS), Brasil - 2017.

\begin{tabular}{|c|c|c|c|c|c|c|}
\hline \multirow{2}{*}{ Variáveis preditoras } & \multirow{2}{*}{ Wald } & \multirow{2}{*}{ df } & \multirow{2}{*}{ Sig } & \multirow{2}{*}{$\operatorname{Exp}(B)$} & \multicolumn{2}{|c|}{$95 \%$ C.I. for $\operatorname{Exp}(\mathrm{B})$} \\
\hline & & & & & Mínimo & Máximo \\
\hline Tempo de trabalho & 0,150 & 1 & 0,040 & 1,612 & 1,143 & 18,117 \\
\hline Treinamento prévio sobre LP & 0,203 & 1 & 0,203 & 1,831 & 0,132 & 25,490 \\
\hline Treinamento prévio sobre DAI & 2,126 & 1 & 0,145 & 4,041 & 0,618 & 28,420 \\
\hline Cargo & 0,126 & 1 & 0,723 & 0,699 & 0,907 & 5,057 \\
\hline Constante & 1,787 & 1 & 0,181 & 0,085 & & \\
\hline
\end{tabular}

LP = Lesão por pressão; DAI = Dermatite Associada à Incontinência.

O modelo não foi estatisticamente significativo ${ }^{2}(4)=, 712 \mathrm{p}=0,607$; Nagelkerke $\left.R^{2}=0,095\right]$, em que apenas o tempo de trabalho maior que 5 anos foi significativo para o conhecimento adequado (Exp(b) 1,612 [95\% IC: 1,143-18,117]), demonstrando que a chance de quem possui mais de 5 anos de experiência apresentar um nível de conhecimento adequado é 1,61 vezes maior que a chance de quem tem menos de 5 anos de experiência apresentar um nível de conhecimento adequado.

\section{DISCUSSÃO}

Considerando os resultados globais deste estudo, verificaram-se médias de acertos significativamente maiores entre os enfermeiros, tanto para a categoria prevenção de LP como para a categoria avaliação e classificação das LP.

Resultados menos satisfatórios foram obtidos em estudos semelhantes com a aplicação do mesmo teste de conhecimento. Em pesquisa realizada com a equipe de enfermagem da Unidade de Terapia Intensiva, verificou-se média total de acertos menor entre os enfermeiros $(51,4 \%)$ do que entre técnicos e auxiliares de enfermagem $(63,4 \%)^{9}$.

Estudo similar realizado no Brasil, com o mesmo instrumento, identificou que apenas 2 enfermeiros $(5,2 \%)$ acertaram $90 \%$ ou mais das questões. A maioria dos enfermeiros acertou entre $70 \%$ e $89 \%$ do instrumento ${ }^{15}$.

Sabe-se que cabe ao enfermeiro privativamente avaliar as lesões e o risco de desenvolvimento de feridas dos pacientes, bem como cabe a esse profissional a prescrição de condutas para a prevenção, tratamento e manejo de lesões de pele, mediante protocolos institucionais. Deste modo, espera-se que os enfermeiros tenham raciocínio clínico e adotem condutas assertivas com relação às lesões de pele e sua prevenção ${ }^{16}$. 
Em relação às medidas de prevenção da LP, houve escores abaixo de $90 \%$ tanto para enfermeiros quanto para os técnicos de enfermagem. Esse achado vai de encontro a outros estudos brasileiros, nos quais foi identificado um conhecimento considerado inadequado quanto à prevenção de LP. Esse dado é preocupante, visto que essa é uma lesão evitável na grande maioria das vezes, podendo ser prevenida com a implementação de cuidados adequados, embasados em evidências científicas, o que vem a ressaltar a importância da implementação de educação permanente sobre esse agravo ${ }^{8,16,17}$.

$\mathrm{Na}$ Turquia, pesquisadores utilizaram o mesmo teste de conhecimento de Pieper, a fim de avaliar o conhecimento de enfermeiros sobre LP. Apenas 8 participantes (2,6\%) pontuaram acima de 80\% e nenhum dos itens foi respondido corretamente por todos os profissionais. A investigação destaca que os escores foram estatisticamente maiores entre enfermeiros que realizam leituras de artigos, participam de cursos relacionados ao tema, cuidam de pacientes com risco de desenvolver ou que possuem $\mathrm{LP}^{18}$.

No presente estudo, verificou-se que os participantes obtiveram uma elevada taxa de acertos, em ambos os grupos profissionais, em 25 questões (61,0\%). Comparativamente, outro estudo brasileiro realizado apenas com enfermeiros, com o mesmo instrumento, evidenciou que apenas 20 itens foram respondidos com uma taxa de acertos acima do desejável ${ }^{15}$.

Foi achado um índice de acertos menor que $50 \% \mathrm{em}$ ambas as categorias profissionais participantes nesse estudo em 5 questões (12,2\%). Essas questões referem-se à espessura da LP de estágio 2, ao uso de luvas d'água para alívio da pressão, ao uso de dispositivos com orifício central para prevenção de LP, ao grau adequado de elevação da cabeceira da cama e à frequência de reposicionamento do paciente enquanto sentado em cadeira de rodas. Outras pesquisas identificaram um baixo índice de acertos no item referente ao uso de almofadas com orifícios centrais para prevenção de LP',15.

Em algumas questões neste estudo foram identificados índices de acertos bem abaixo do esperado em ambas as categorias profissionais. Um desses itens está relacionado à aplicação de massagens em regiões de proeminências ósseas. Em estudos semelhantes foi verificado um índice de acertos bem abaixo do adequado dos participantes nesse mesmo item ${ }^{9,10}$.

Sabe-se que há duas décadas, nos Estados Unidos da América, ocorreu a publicação de uma diretriz que contraindicava o uso de luvas d'água, bolsas de soro e/ou almofadas com orifícios centrais para prevenção da LP. Nesse documento era ressaltado que a realização de massagens em regiões de proeminências ósseas não deveria ser realizada devido ao risco desse procedimento piorar a congestão local, aumentando, assim, o risco de surgimento de uma LP. Essas orientações estão divulgadas em documentos mais recentes, inclusive traduzidos para a língua portuguesa ${ }^{8}$.

Neste contexto, ressalta-se o importante papel da educação permanente dentro das instituições de saúde como agente de mudanças das práticas de cuidado. A educação permanente é realizada no serviço e para o serviço, ou seja, é voltada para atender as dúvidas dos servidores frente às patologias encontradas no dia a dia de seu trabalho em saúde. Essa educação deve ser vislumbrada como prioridade dentro das instituições, com o intuito de promover melhorias no cuidado prestado ao cliente ${ }^{19-22}$.

No Brasil, as medidas relacionadas aos protocolos de prevenção e tratamento no que concerne às ações educativas são recentes e começaram a ser instituídas devido aos credenciamentos institucionais para a qualidade e segurança do paciente ${ }^{2,9}$.

A presente pesquisa possui algumas limitações, dentre elas está o tamanho amostral reduzido. Além disso, a equipe multiprofissional não foi incluída e não foi realizada a comparação do grau de conhecimento dos respondentes antes e após a realização de atividade de intervenção educativa. Devido ao período da coleta de dados, outra limitação está relacionada ao instrumento utilizado, visto que no Brasil já existe um instrumento mais recente e validado para a mesma finalidade ${ }^{4}$ Recomenda-se a realização de estudos semelhantes, multicêntricos, com o intuito de averiguar o cenário nacional sobre o conhecimento de profissionais de enfermagem acerca da prevenção, avaliação e classificação da LP.

\section{CONCLUSÃO}

Neste estudo foi possível identificar lacunas de conhecimento entre 50 profissionais de enfermagem de uma unidade de clínica médica de um hospital universitário, considerando que a média de acerto global referente às 41 questões foi menor que $90 \%$.

Com o intuito de melhorar o conhecimento sobre esse assunto, as instituições de assistência à saúde devem implementar ações de educação permanente acerca desse agravo, tendo em vista os custos que acarretam e suas complicações. Esses 
processos educacionais devem ser pautados no diálogo para favorecer a troca de experiências e aumentar o sentimento de pertencimento aos envolvidos. O uso de estratégias educativas diversificadas, como roda de conversa, materiais impressos, vídeos, entre outros, podem ser utilizados com o objetivo de favorecer a aprendizagem dos participantes.

\section{CONTRIBUIÇÃO DOS AUTORES}

Conceitualização: Sokem JAS, Bergamaschi FPR e Watanabe EAMT; Metodologia: Sokem JAS, Bergamaschi FPR, Watanabe EAMT, Ferreira AM, Siqueira LDC e Coelho MMF; Investigação: Sokem JAS, Bergamaschi FPR e Watanabe EAMT; Redação - Primeira versão: Sokem JAS, Bergamaschi FPR, Watanabe EAMT, Ferreira AM, Siqueira LDC e Coelho MMF; Redação - Revisão \& Edição: Sokem JAS, Bergamaschi FPR, Watanabe EAMT, Ferreira AM, Siqueira LDC e Coelho MMF; Recursos: Sokem JAS, Bergamaschi FPR e Watanabe EAMT; Supervisão: Bergamaschi FPR e Watanabe EAMT.

\section{DISPONIBILIDADE DE DADOS DE PESQUISA}

Todos os dados foram gerados ou analisados no presente estudo.

\section{REFERÊNCIAS}

1. Sassaki RL, Cucolo DF, Perroca MG. Interruptions and nursing workload during medication administration process. Rev Bras Enferm. 2019;72(4):1001-6. http://doi.org/10.1590/0034-7167-2018-0680

2. Agência Nacional de Vigilância Sanitária (BR). Nota técnica GVIMS/GGTES n 03/2017: Práticas seguras para prevenção de Lesão por Pressão em serviços de saúde. Brasília (DF); 2017 [acesso em 30 jul 2021]. Disponível em: https://www.gov.br/ anvisa/pt-br/centraisdeconteudo/publicacoes/servicosdesaude/notas-tecnicas/nota-tecnica-gvims-ggtes-no-03-2017.pdf

3. National Pressure Ulcer Advisory Panel. National Pressure Ulcer Advisory Panel Responds to Editorial. Adv Skin Wound Care. 2016;29(12):535. https://doi.org/10.1097/01.ASW.0000508227.61503.f9

4. Rabeh SAN, Palfreyman S, Souza CBL, Bernardes RM, Caliri MHL. Cultural adaptation of the Pieper-Zulkowski Pressure Ulcer Knowledge Test for use in Brazil. Rev Bras Enferm. 2018;71(4):2092-100. https://doi.org/10.1590/0034-7167-2017-0029

5. Barbosa JM, Salomé GM. Occurrence of pressure injury in patients hospitalized in a school hospital. ESTIMA, Braz.J. Enterostomal Ther. 2018;16:e2718. https://doi.org/10.30886/estima.v16.523

6. Kayser SA, VanGilder CA, Ayello EA, Lachenbruch C. Prevalence and Analysis of Medical Device-Related Pressure Injuries: Results from the International Pressure Ulcer Prevalence Survey. Adv Skin Wound Care. 2018;31(6):276-85. https://doi.org/10.1097/01. ASW.0000532475.11971.aa

7. Rabeh SAN, Gonçalves MBB, Caliri MHL, Nogueira PC, Miyazaki MY. Terapia tópica para heridas crónicas: contribuciones de un módulo de enseñanza a distancia para el conocimiento de estudiantes de enfermería. Enfermería Global. 2017;16(1):69-101. https://doi.org/10.6018/eglobal.16.1.237361

8. National Pressure Ulcer Advisory Panel; European Pressure Ulcer Advisory Panel; Pan Pacific Pressure Injury Alliance. Prevention and Treatment of Pressure Ulcers: Quick Reference Guide. Emily Haesler (Ed.). Cambridge Media: Osborne Park (Aus); 2019. Disponível em: https://www.epuap.org/download/11182/

9. Galvão NS, Serique MAB, Santos VLCG, Nogueira PC. Knowledge of the nursing team on pressure ulcer prevention. Rev Bras Enferm. 2017;70(2):294-300. https://doi.org/10.1590/0034-7167-2016-0063

10. De Meyer D, Verhaeghe S, Van Hecke A, Beeckman D. Knowledge of nurses and nursing assistants about pressure ulcer prevention: A survey in 16 Belgian hospitals using the PUKAT 2.0 tool. J Tissue Viability. 2019;28(2):59-69. https://doi. org/10.1016/j.jtv.2019.03.002

11. Charalambous C, Koulouri A, Roupa Z, Vasilopoulos A, Kyriakou M, Vasiliou M. Knowledge and attitudes of nurses in a major public hospital in Cyprus towards pressure ulcer prevention. J Tissue Viability. 2019;28(1):40-45. https://doi.org/10.1016/j. jtv.2018.10.005

12. Damme NV, Hecke AV, Himpens A, Verhaeghe S, Beeckman D. Design and psychometric testing of the attitude towards the prevention of incontinence-associated dermatitis instrument (APrIAD). Int Wound J. 2019;16(2):492-502. https://doi. org/10.1111/iwj.13062 
13. Pieper B, Mott M. Nurses' knowledge of pressure ulcer prevention, stating, and description. Adv Wound Care. 1995 [acesso em 05 ago 2021];8(3):34-48. Disponível em: http://europepmc.org/abstract/med/7795877

14. Fernandes LM, Caliri MHL, Haas VJ. The effect of educative interventions on the pressure ulcer prevention knowledge of nursing professionals. Acta Paul Enferm. 2008 [acesso em 21 jul 2021];21(2):305-11. Disponível em: http://www2.unifesp.br/ acta/pdf/v21/n2/v21n2a12.pdf

15. Sousa RC, Faustino AM. Conhecimento de enfermeiros sobre prevenção e cuidados de lesão por pressão. Fundam Care Online. 2019;11(4):992-7. http://doi.org/10.9789/2175-5361.2019.v11i4.992-997

16. Albuquerque AM, Vasconcelos JMB, Souza APMA, Chaves TRCL, Costa IKF, Soares MJGO. Knowledge test on pressure injury. Rev enferm UFPE on line. 2018;12(6):1738-50. https://doi.org/10.5205/1981-8963-v12i6a234578p1738-1750-2018

17. Portugal LBA, Chirstovam BP, Mendonça RP. The nurses' knowledge about pressure injury care. Revista Enfermagem Atual. 2018;84(22):59-68. http://.doi.org/10.31011/1519-339X.2018a18n84.5

18. Gul A, Andsoy II, Ozkaya B, Zeydan A. A Descriptive, Cross-sectional Survey of Turkish Nurses' Knowledge of Pressure Ulcer Risk, Prevention, and Staging. Ostomy Wound Manage. 2017 [acesso em 04 ago 2021];63(6):40-46. Disponível em: https:// www.o-wm.com/article/descriptive-cross-sectional-survey-turkish-nurses-knowledge-pressure-ulcer-risk-prevention

19. Ceccim RB. Emergência de um "campo de ação estratégica": ordenamento da formação e educação permanente em saúde. SANARE. 2019 [acesso em 07 set 2021];18(01):68-80. Disponível em: https://sanare.emnuvens.com.br/sanare/article/ download/1307/688

20. Lavich CRP, Terra MG, Mello AL, Raddatz M, Arnemann CT. Ações de educação permanente dos enfermeiros facilitadores de um núcleo de educação em enfermagem. Rev Gaúcha Enferm. 2017;38(1):e62261. http://doi.org/10.1590/19831447.2017.01.62261

21. Flores GE, Oliveira DLL, Zocche DAA. Permanent education in the hospital context: the experience that brings new meaning to nursing care. Trab. Educ. Saúde. 2016;14( 2):487-504. http://doi.org/10.1590/1981-7746-sip00118

22. Inagaki ADM, Souza IES, Araujo ACL, Abud ACF, Cardoso NP, Ribeiro CJN. Conhecimento de médicos e enfermeiros atuantes no pré-natal sobre toxoplasmose. Cogitare enferm. 2021;26:e70416. http://doi.org/10.5380/ce.v26i0.70416 